\title{
The ambiguous sea level rise at Brest's 212 yearlong record elucidated
}

DOI: https://doi.org/10.1515/jogs-2020-0124

Received January 20, 2021; accepted July 1, 2021

\begin{abstract}
The tide gauge record at Brest, France, along Eastern part of Atlantic coast is one of the longest records in Europe spanning 212 years (1807-2019). Analyzing these records has important ramifications in assessing anthropogenic impact of climate change at local and regional scales during this period. All the previous studies that analyzed Brest's tide gauge record have used vaguely defined quadratics models and did not incorporate the effect of sea level variations at various frequencies, which confounded the presence or absence of a plausible uniform acceleration. Here, we entertained two competing kinematic models; one with a uniform acceleration representing 212 years of monthly averaged tide gauge data, the other is a twophase trend model (Phase I is 93 years long and Phase II is 119 years long). Both models include statistically significant ( $\alpha=0.05)$ common periodic effects, and sub and super harmonics of luni-solar origin for representing monthly averaged sea level anomalies observed at Brest. The least squares statistics for both models' solutions cannot distinguish one model over the other, like earlier studies. However, the assessment of Phase I segment of the records disclosed the absence of a statistically significant trend and a uniform acceleration during this period. This outcome eliminates conclusively the occurrence of a uniform acceleration during the entire 212-year data span of the tide gauge record at Brest, favoring the two-phase trend model as a sound alternative.
\end{abstract}

Keywords: Brest tide gauge; Mean sea level trend; Uniform sea level acceleration; Two phase trend; Climate change

The idea is that ambiguity involves different interpretations while vagueness involves different ways in which a given interpretation can be made precise.

Kees Van Deemter, 2010.

H. Bâki İz: Division of Geodetic Science, School of Earth Sciences, The Ohio State University Columbus, Ohio, USA

C.K. Shum: Division of Geodetic Science, School of Earth Sciences, The Ohio State University Columbus, Ohio, USA

\section{Introduction}

Evidence for mean sea level rising faster during the 19th, 20th, and 21st centuries with warming is an important indicator in assessing anthropogenic contributions to the climate change mechanisms. Although there are several tide gauges (TG) available for this purpose, only few of them span a lengthy period to be useful in accounting for the confounding effect of long periodic (LP) ${ }^{1}$ sea level variations in detecting sea level accelerations. Brest, France, TG station is located at the western extremity of France, bordering Atlantic Ocean and has one of the longest records in Europe spanning 212 years (1807-2019). The data set is available at the Permanent Service for Mean Sea Level (PSMSL) repository.

Among several studies that investigated sea level accelerations around the globe using TGs , Woodworth et al. (1990) reported uniform sea level decelerations $-0.022 \pm$ 0.036 during $1930-1985$ and $-0.003 \pm 0.007 \mathrm{~mm} / \mathrm{yr}^{2}$ during $1870-1985$. Both estimates cannot reject the null hypothesis (no acceleration) at 5\% significance level ${ }^{2}(\alpha=0.05)$. In a follow up study, Woodworth et al. (2011) reported $0.003 \pm 0.001 \mathrm{~mm} / \mathrm{yr}^{2}$ uniform sea level acceleration during 1750-2010 at Brest that rejects the null hypothesis. More recently, Boretti (2020) estimated an average ${ }^{3}$ sea level acceleration of $0.010 \pm 0.005 \mathrm{~mm} / \mathrm{yr}^{2}$ using a quadratic model (akin to a uniform acceleration) at Brest during 1857-2018, which is borderline for rejecting the null hypothesis of a uniform acceleration.

One of the investigations by Gornitz and Solow (1991) identified a change point during 1897 at this station and used a two-phase regression model ${ }^{4}$ that was deployed earlier by Solow (1987) for climate change studies. Their

\footnotetext{
1 Long periodic sea level changes refer to a broad range of periodicities including interannual, decadal, interdecadal and longer in this study.

2 We will use $\alpha=0.05$ criterion for the statistical significance assessment throughout the manuscript.

3 This is a misnomer since acceleration estimated from a quadratic model is a uniform acceleration. Average acceleration is, by definition, the change in velocity during a period.

4 They did not report trend estimates for their two-phase model.
} 
conclusion was that it is unlikely the rate of $\mathrm{SL}^{5}$ rise change suddenly. Another study by Solow (1990) using the same two-phase and a quadratic model concluded that it was impossible to discriminate between the quadratic model and the two-phase model. From a practical point of view, this means that it is unclear whether the apparent acceleration in relative sea level at Brest occurred over a relatively short period of time near the turn of the century or whether it occurred smoothly over the course of the data.

Wöppelman et., all (2006) estimated a sea level acceleration, $0.007 \pm 0.001 \mathrm{~mm} / \mathrm{y} 2$ during 1807-2004 using a quadratic model. They also identified variable trends during 1807-1890, 1890-1980, and 1980-2004. Most recently, Iz et al. (2018) estimated a statistically significant average acceleration $0.024 \pm 0.003 \mathrm{~mm} / \mathrm{yr}^{2}$ (not the uniform acceleration) using all the available data at PSMSL database for this station together with the satellite altimetry data starting 1993 onward with a two-phase trend model. At this point, it should be noted that common to all studies, other than by Iz et al. (2018), ignored taking into account the effect of LP sea level variations at Brest in their models. The aforementioned studies investigating the sea level acceleration at Brest are not exhaustive, but they already reveal inconsistent and vague acceleration estimates leaving the nature of the sea level rise issue at this important station unsettled.

In the following sections, we first give a brief description of the TG data at Brest, France. We then set up two competing models inspired by the previous studies; a kinematic model with a secular trend and uniform acceleration, and a two-phase trend model to represent SL variations. Both models include LP sea level representations. Ordinary Least Squares (OLS) estimates for the model parameters are then reported together with other solution statistics followed by a discussion on the nature of sea level rise at Brest during 1807-2019.

\section{Tide Gauge Records at Brest}

Brest monthly TG time series data displayed in Figure 1 were downloaded from the PSMSL repository on September 2020 (PSMSL, 2020). These series were extensively scrutinized by Wöppelmann et al. (2006, 2008). All records are referenced to the Revised Local Reference (RLR). No corrections including post glacial rebound, nor inverted barometric (IB) effects were applied to the data. Therefore, all secular estimates are relative trends and the effect of the

5 SL refers to sea-level.
IB is accounted for through the sub and super harmonics incorporated into the models as they are discussed in the subsequent sections. One visually notable property of the series is the sharp change that occurred around 1900 as can be seen from the smoothed series displayed in Figure 1. In the following sections, we will establish and analyze the two competing kinematic models to analyze the Brest tide gauge record.

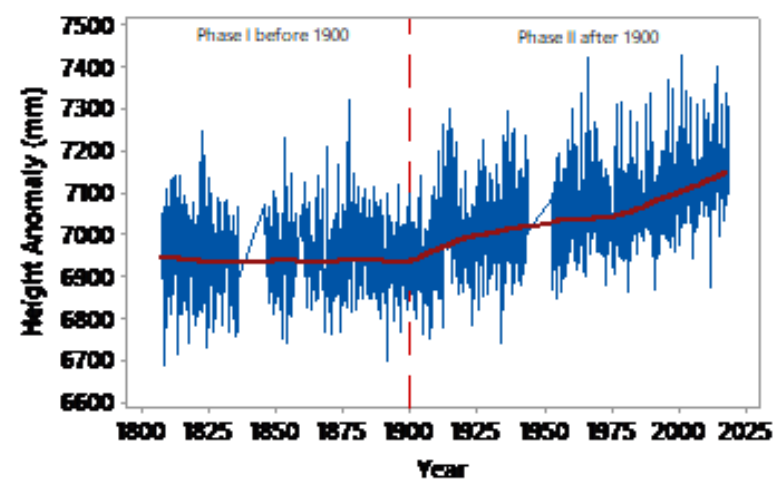

Fig. 1. Monthly averaged sea level height anomalies and their smoothed (Lowess) values at Brest.

\subsection{Selective Nomenclature}

The analyses reported in the following sections rely crucially on the precise definitions of the following terms in the context of sea level measurements.

A quadratic function is frequently used in sea level studies and known in algebra as a quadratic polynomial or a polynomial of degree 2 or simply quadratic,

$$
f(t)=\alpha+\beta t+y t^{2}, y \neq 0
$$

where $\alpha, \beta, y$ are constants.

Uniform acceleration, also known as constant acceleration, is defined as the rate of change in velocity,

$$
v=v_{0}+a t, \Rightarrow a=\frac{v-v_{0}}{t} \neq 0
$$

where $a, v_{0}$ and $v$ are the uniform acceleration, initial velocity at a predefined epoch $t_{0}$, and velocity at an arbitrary epoch $t$ respectively. For the vertical displacement, $h$, this expression leads to,

$$
h=h_{0}+v_{0} t+\frac{a}{2} t^{2}
$$

This model is a kinematic representation of the sea level change. Comparing eqn. (3) with eqn. (1) reveals the 
equivalence of quadratics with the uniform acceleration model for which,

$$
v_{0}=\beta, a=2 y \Rightarrow \sigma_{a}=2 \sigma_{y}
$$

where $\sigma_{a}, \sigma_{y}$ are their corresponding standard deviations if they are to be estimated. It is important to note that $\beta$ represented by the quadratic is the initial velocity $v_{0}$ at $t_{0}$ and will be different than the velocity at $t$, i.e., $v_{0} \neq v$, when $a \neq 0$. This distinction necessitates that if two different velocities are to be compared, their initial epochs must be the same. The requirement disappears when $a=0$.

Average velocity $\hat{v}$ is, by definition, the change in the vertical direction $\Delta h$ over a period $\Delta t$, i.e.

$$
\hat{v}:=\frac{\Delta h}{\Delta t}=\frac{v_{0}+v}{2} \neq v \neq v_{0}, a \neq 0
$$

Average acceleration $\hat{a}$ is the change in the velocity $\Delta v$ over a period $\Delta t$,

$$
\hat{a}:=\frac{\Delta v}{\Delta t} \neq a, a \neq 0
$$

With these definitions in consideration, we model the sea level variations at Brest in the following section.

\section{Kinematic model with a trend, uniform acceleration and periodicities at Brest}

The first of the two models that represents the observed sea level height anomalies consisting of a trend (initial velocity), uniform acceleration, and periodic sea level variations is as follows,

$$
\begin{aligned}
& h_{t}=h_{1900}+v_{0}(t-1900)+\frac{a}{2}(t-1900)^{2} \\
& +\sum_{k=1}^{17}\left[\alpha_{k} \cos \left(\frac{2 \pi}{P_{k}}\right)(t-1900)+y_{k} \sin \left(\frac{2 \pi}{P_{k}}\right)(t-1900)\right] \\
& +\epsilon_{t}
\end{aligned}
$$

In this representation, an observation at an epoch $t$ is denoted by $h_{t}, t=1 \cdots n$, where $n$ is the total number of monthly averaged TG measurements. The intercept $h_{1900}$ is the height of the sea level defined at the reference epoch year 1900 during which there may be a sharp change in sea level trend as implicated by the smoothed anomalies in Figure 1 . The initial velocity at $t_{1900}$ when $a \neq 0$, is denoted by $v_{0}$, and $a$ is the constant rate of change in sea level velocity (i.e., uniform acceleration).
What is markedly different in this model as compared to the previous studies is modelling various LP sea level variations. For instance, Jevrejeva, et al., (2006) reported nonlinear trends and multiyear cycles in sea level records up to 30 years. The origins of these global oscillations are multi-causal. The interactions of the deterministic components of sea level variations with those from natural ocean and atmosphere interactions are suggested by Munk et al. (2002) and Keeling and Whorf (1997). Under their scenarios, interactions of ocean, meteorological forcing, atmospheric, and sea surface temperature materialize as natural broad band sea level variations. They modulate the effect of astronomical forcing, such as lunar node tide and or random beatings resulting in sub and super harmonics of known periods (Table 1). Similarly, the variations in total solar radiation with a period of $\mathrm{P}=11.1 \mathrm{yr}$., yield subharmonics with periods: $2 \times \mathrm{P}=22.2 \mathrm{yr}$. and longer.

The compounding of sea level periodic variations and their materializations in sea level variations at multidecadal scales is not a conjecture. Yndestad et al. (2008), for instance, reported that the water-property time-series show mean variability correlated to a subharmonic cycle of the nodal tide of about 74 years. They found significant correlations between dominant Atlantic water temperature cycles and the 18.6-year lunar node tide, and for $P / 2=$ 9.306-year lunar nodal phase tide. An earlier wavelet analysis by Yndestad (2006) identified a number of lunar node sub and super harmonics in Arctic Sea level, temperature, ice extent and winter index time series data, including the signature of nodal harmonics in pole position time series (Table 1 in Yndestad, 2008), and a strong cross correlation with Chandler wobble.

Although the observed amplitudes of the 18.6-year nodal constituent are small, amounting $\sim 15-35 \mathrm{~mm}$, compounding of the nodal tide with natural sea level variations can confound sea level trend and acceleration estimates particularly for short TG and SA time series and thereby hinder the search of a global GMSL acceleration caused by anthropogenic global warming. As early as 2006, İz has quantified theoretically that these periodicities despite their small amplitudes, if not modelled, will bias the sea trend estimates for shorter series. Moreover, their effects may also be mistakenly interpreted as an accelerated sea level rise if they are not incorporated into the models in analyzing short as well as longer series. İz (2014) study revealed statistically significant periodic oscillations at $27 \mathrm{TG}$ stations with long records. In addition, the follow up study by İz (2015) demonstrated that once these effects are modeled and the corresponding model parameters are estimated, spectral analysis of the TG residuals reveals additional statistically significant sea level 
variations at decadal scale. İz (2018) study attributed these changes due to the ocean surface wind forcings and periodic changes in atmospheric pressure, inverted barometer (IB) effect along the coastal lines of some TG stations and quantified their periodicities. Among them, 12.4 yr. periodicity was prominent and statistically significant at Brest TG station. Hence it is incorporated also into the current model.

The intent of including these periodicities into the kinematic model is to prevent biasing the estimated parameters (confounding). They also reduce the SEs of the solutions and the SEs of the estimated parameters for assessing their statistical significances effectively.

In this model, the periodicities, $P_{k}, k=1 \cdots m$ where $m$ include a mix of seventeen sub and super harmonics attributed to the node tides solar radiation, annuals and sub annuals shown in Table 1 and 12.4-year periodicity caused by the IB effects and currents at this location. Each period introduces two parameters, $\alpha_{k}, y_{k}$ for the sine and cosine components from which the amplitudes $a_{k}$ and the phase angles of the periodic terms are determined. In total, the extended kinematic model includes 37 unknown parameters.

As far as the statistical properties of the model is concerned, the disturbances are independently, randomly, and homogeneously distributed, i.e., $\epsilon_{t} \sim\left(0, \sigma_{\epsilon}^{2}\right)$, where $\sigma_{\epsilon}^{2}$ is the variance of the disturbances. The square root of its estimate is denoted by the standard error of the solution, $\hat{\sigma}_{\epsilon}$ or simply SE. First order autocorrelation AR(1), which is present with varying magnitudes in globally distributed tide gauge stations (Iz, et. al., 2012) is about $\hat{\rho}=0.1$ for Brest record once the LP sea level variations are modeled. Therefore, the autocorrelation can be safely ignored in this study. However, because the LP sea level variations were not modeled in previously reported studies, the magnitude of the AR(1) autoregressive correlation coefficient was large, $\hat{\rho}=0.4$. This omission error reduced the statistically effective length of the total series ${ }^{6}$, hence impacted all the pertinent statistics of the previously reported estimates adversely (Type I error).

In the following section, we consider a competing model to represent sea level anomalies at Brest.

6 For this study, the effective length of the series is reduced from 212 yr. down to $91 \mathrm{yr}$. if LP sea level variations are not modeled.

\section{A two-phase trend model with common uniform acceleration}

A strip-down version of the model that is presented in this section was also entertained by Solow (1990), and Gornitz and Solow (1991), without modeling LP sea level anomalies. It is important to note again that modelling LP sea level variations are important to prevent biasing the uniform acceleration and trend estimates. Their inclusion also increases the statistical power of the solutions (Adj. $R^{2}$ values) by reducing the SE of the solution (akin to, the a posteriori variance of the unit weight) and the SE of the estimates, which are vital in testing the null hypotheses.

We formulate a two-phase trend model as follows,

$$
\begin{aligned}
& h_{t}=h_{1900}+v_{0}{ }^{P H I}\left(t^{P H I}-1900\right) \\
& +v_{0}{ }^{P H I I}\left(t^{P H I I}-1900\right)+\frac{a}{2}(t-1900)^{2} \\
& +\sum_{k=1}^{37}\left[\alpha_{k} \cos \left(\frac{2 \pi}{P_{k}}\right)(t-1900)+y_{k} \sin \left(\frac{2 \pi}{P_{k}}\right)(t-1900)\right] \\
& +\epsilon_{t}
\end{aligned}
$$

This model is similar to the previous one with the emphasis on two-trend representations in two phases denoted by Phase I, and Phase II that occurs after 1900 as revealed graphically in Figure 1. This date is also the common initial period for the trends and uniform acceleration parameter all referring to the same datum $h_{1900}$. The statistical properties of the disturbances and modeled periodic variations are the same as in the first model.

\subsection{Solutions and discussion}

The unknown model parameters of both models were estimated (Table 2 and Table 3) using OLS. The statistically significant components of the estimated periodicities tabulated in Table 3 are common in both models and they do not differ significantly from model to model.

In Figure 2, the solution residuals are displayed against the adjusted sea level anomalies together with their histograms with overlapping normal distributions. The plots validate that the residuals are random in nature, free from any pronounced systematic unmodeled effects. Both model solutions exhibit similar distributional properties, which are testament for the elusive nature of the sea level variations at this station.

However, the estimated initial velocity and uniform acceleration parameters of the first model are statistically significant. The model explains $51.3 \%$ of the variation in 
Table 1. Compounded Luni-Solar periods (yr.).

\begin{tabular}{l|l|l|l|l|l}
\hline $\begin{array}{l}\text { Nodal } \\
\text { Subharmonics }\end{array}$ & $\begin{array}{l}\text { Nodal } \\
\text { Superharmonics }\end{array}$ & $\begin{array}{l}\text { Nodal } \\
\text { Superharmonics }\end{array}$ & Solar & $\begin{array}{l}\text { Annual\& } \\
\text { Subannuals }\end{array}$ & Chandler \\
\hline 74.5 & 18.6 & 3.7 & 11.1 & 1.00 & $429.5 / 365.4$ \\
55.8 & 9.3 & 3.1 & 22.2 & 0.50 & \\
37.2 & 6.2 & 2.6 & & 0.25 & \\
& 4.7 & 2.3 & & & \\
\hline
\end{tabular}

Table 2. OLS solution statistics. Trend and uniform acceleration estimates in bold are statistically significant at $\alpha=0.05$.

\begin{tabular}{l|l|l|l|l|l|l}
\hline Model & $\begin{array}{l}\text { Time Span } \\
\text { yr. }\end{array}$ & $\begin{array}{l}\text { Initial } \\
\text { Epoch } \\
\text { yr. }\end{array}$ & $\begin{array}{l}\text { Initial Ve- } \\
\text { locity } \\
\mathrm{mm} / \mathrm{yr} .\end{array}$ & $\begin{array}{l}\text { Uniform Ac- } \\
\text { celeration } \\
\mathrm{mm} / \mathrm{yr}^{2}\end{array}$ & $\begin{array}{l}\text { SE } \\
\mathrm{mm}\end{array}$ & $\begin{array}{l}\text { Adj } \mathbf{R}^{2} \\
\%\end{array}$ \\
\hline $\begin{array}{l}\text { Uniform Acceleration } \\
\text { + Trend }\end{array}$ & $\begin{array}{l}1807-2019 \\
212\end{array}$ & 1900 & $\mathbf{0 . 7 8 \pm 0 . 0 3}$ & $\mathbf{0 . 0 1 3 \pm 0 . 0 0 1}$ & $\mathbf{7 5 . 3}$ & 51.3 \\
\hline $\begin{array}{l}\text { Uniform Acceleration + } \\
\text { Two-phase Trend }\end{array}$ & $\begin{array}{l}1807-1900 \\
93\end{array}$ & 1900 & $\mathbf{0 . 0 5 \pm 0 . 0 7}$ & $\mathbf{0 . 0 0 4 \pm 0 . 0 0 3}$ & 75.2 & 51.4 \\
& $\begin{array}{l}1900-2019 \\
119\end{array}$ & & $1.60 \pm 0.04$ & & & \\
\hline
\end{tabular}

Table 3. The estimated statistically significant components of periodicities and their SEs. $\mathrm{C}$ is for cosine, $\mathrm{S}$ is for the sine component of a periodicity and the concatenated numbers are the rounded periods in years given in Table 1. Units are in $\mathrm{mm}$.

\begin{tabular}{l|l|l|l|l|l|l|l|l|l|l|l}
\hline C75 & C37 & S18.6 & S12.4 & S11 & C9 & C6 & S4 & C2.6 & Sann & Cann & Ssemi \\
\hline $\mathbf{- 1 0 . 4 2}$ & -9.76 & -7.97 & 6.00 & 5.04 & -8.45 & -5.04 & -4.91 & 5.43 & -32.93 & 37.5 & -19.7 \\
\hline \pm 2.23 & \pm 2.26 & \pm 2.30 & \pm 2.28 & \pm 2.26 & \pm 2.26 & \pm 2.22 & \pm 2.23 & \pm 2.23 & \pm 2.23 & \pm 2.23 & \pm 2.23 \\
\hline
\end{tabular}

Table 4. OLS solution statistics using uniform acceleration model. Trend and velocity estimates in bold are statistically significant at $\alpha=0.05$. Phase I parameters were estimated using year 1807 and 1900 to check the robustness of the LP components. Note that the initial velocity is dependent on the inial epoch but the uniform acceleration is invariant with repect to the choice of the initial epoch. Initial velocity estimate in bold is statistically significant.

\begin{tabular}{l|l|l|l|l|l|l}
\hline $\begin{array}{l}\text { Solution } \\
\text { Uniform } \\
\text { acceleration model) }\end{array}$ & $\begin{array}{l}\text { Time Span } \\
\text { yr. }\end{array}$ & $\begin{array}{l}\text { Initial } \\
\text { Epoch } \\
\text { yr. }\end{array}$ & $\begin{array}{l}\text { Initial Ve- } \\
\text { locity } \\
\mathrm{mm} / \mathrm{yr} .\end{array}$ & $\begin{array}{l}\text { Uniform Acceler- } \\
\text { ation } \\
\mathrm{mm} / \mathrm{yr}^{2}\end{array}$ & $\begin{array}{l}\text { SE } \\
\mathrm{mm}\end{array}$ & $\begin{array}{l}\text { Adj. } \\
\mathbf{R}^{2} \\
\%\end{array}$ \\
\hline $\begin{array}{l}\text { Phase I } \\
\text { Independent }\end{array}$ & $\begin{array}{l}1807-1900 \\
93\end{array}$ & 1900 & $-0.52 \pm 0.36$ & $-0.010 \pm 0.007$ & 72.6 & 22.5 \\
\hline Phase II Independent & $\begin{array}{l}1900-2019 \\
119\end{array}$ & 1900 & $1.22 \pm 0.25$ & $0.004 \pm 0.004$ & 76.2 & 44.9 \\
\hline
\end{tabular}

the series with a SE of $75.3 \mathrm{~mm}$. Shown on the same table are the two-phase trend model's initial trend estimates, which are constant velocities in sea level rise (uniform acceleration is rejected). It is important to note that the trends estimated in these two models are of different nature. The first model's estimated trend is the initial velocity $\hat{v}_{0}$ but the velocity varies throughout the 212-year series because the acceleration is statistically significant. Whereas the trends estimated in the second model are constant over time during Phase I and Phase II time span because of the absence of a statistically significant acceleration during these periods (overlapping uniform acceleration estimate is not statistically significant). Notably, such distinc- tions have not been made in the past and in the current literature causing ambiguity and vagueness in reported sea level accelerations around the globe. In addition, the null hypothesis for the estimated constant velocity for Phase I cannot be rejected at $\alpha=0.05$ significance level. The second model explains $51.4 \%$ of the variation in the series with a SE $=75.2 \mathrm{~mm}$. The trend of Phase I, $0.05 \pm 0.07 \mathrm{~mm} / \mathrm{yr}$., and the uniform acceleration $0.004 \pm 0.003 \mathrm{~mm} / \mathrm{yr}^{2}$ for the same period are small in magnitude, and the null hypotheses for their statistical significance cannot be rejected. And finally, given the similarities in magnitudes of the solution SEs and the $A d j . R^{2}$ values, both models seem to be indistinguishable hence support the conclusions reached 

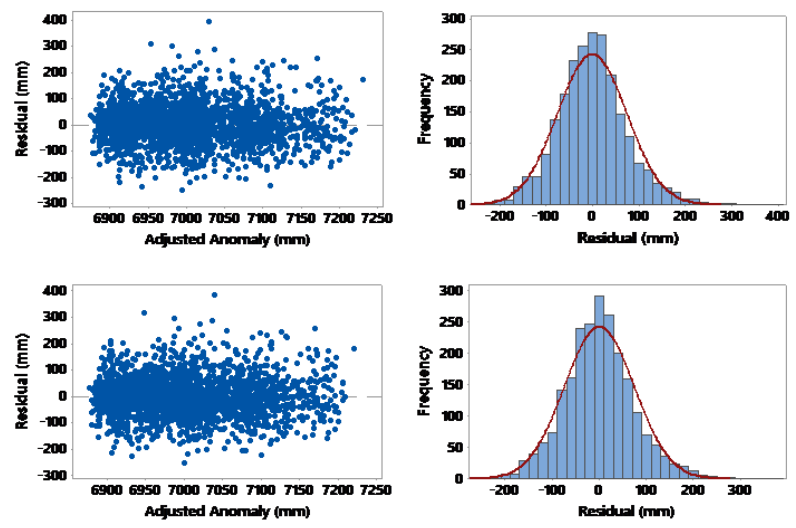

Fig. 2. Residual distributions for the uniform acceleration and trend model (top two plots) and uniform accleration with two-phase trend model. The residuals plotted against the adjusted height anomalies and histograms are indicative of their randomness, free from unmodeled systematic effects.

by Solow (1990), and Gornitz and Solow (1991) 30 years ago despite the improved models to mitigate statistically significant LP periodic variations at this station. The residuals and their distributional properties shown in Figure 1 also support the ambiguity.

At this point, we conducted two additional separate OLS solutions using the uniform acceleration model for the Phase I and Phase II records sharing the same initial epoch year 1900 and 1807 as the initial epoch for verification. The SE of each solution listed in Table 4 show that the initial velocity and uniform acceleration estimates are not statistically significant for the Phase I solution and only the initial velocity turned out to be statistically significant for the Phase II solution. The SE of the Phase I solution is smaller that the Phase II solution hinting the homogeneity of the measurements throughout the series, whereas the $A d j . R^{2}$ value for the Phase I solution was markedly small (Adj. $R^{2}=22.5 \%$ ) because there is no signal for the model to explain once the LP sea level variations are accounted for. But this is exactly what the Phase I solution unravels: the absence of a statistically significant initial velocity and a uniform acceleration. This standalone finding is necessary and sufficient to eliminate the uniform acceleration model based on the crisp definition of the uniform velocity. The uniform acceleration model that encompasses 212 years cannot be the correct model since, by definition, the uniform acceleration must be the same throughout the time series, but it is absent during the Phase I.

\section{Conclusion}

We replicated the enigmatic findings of an ambiguous sea level rise at Brest published three decades ago. A closer inspection of the initial velocity and uniform acceleration estimates at Brest that were both absent during the Phase I period effectively eliminated the possibility of a uniform acceleration during the 212-year timespan of the series. This finding was due to the improvements in the solution statistics because of modelling and estimating statistically significant periodic variations, which successfully reduced the vagueness of the underlying kinematics of the sea level variations at this station. This observational evidence provided an unambiguous answer to the kinematics of the sea level rise at this station but tells nothing about the underlying cause of the sudden increase in sea level since 1900.

Acknowledgments: We would like to thank the two reviewers for their time and helpful comments. This research is partially supported by Natural Science Foundation of China (41584016, 41974040).

\section{References}

Boretti, A. 2020, The pattern of sea-level rise across the North Atlantic from long-term-trend tide gauges. Ocean \& Coastal Management, Vol., 196.

Gornitz, V. and Solow, A., 1991, Observations of Long-Term TideGauge Records for Indicators of Accelerated Sea Level Rise, in Schlesinger, M. E. (ed.),Greenhouse Gas-Induced Climatic Change: A Critical Appraisal of Simulations and Observations, Elsevier, Amsterdam, pp. 347-367.

İz H.B., C.K. Shum, C.Y. Kuo, 2018, Sea Level Accelerations at Globally Distributed Tide Gauge Stations During the Satellite Altimetry Era, J. Geod. Sci. Vol. 8, pp. 130-135.

İz H.B., 2018, The effect of regional sea level atmospheric pressure to sea level variations at globally distributed tide gauge stations with long records, J. Geod. Sci. Vol. 8, pp. 55-71.

İz H.B., 2015, More confounders at global and decadal scales in detecting recent sea level accelerations, J. of Geod. Sci, 5, 192198.

İz H.B., 2014, Sub and super harmonics of the lunar nodal tides and the solar radiative forcing in global sea level changes, J. of Geod. Sci., 4, 150-165.

İz H.B., L. Berry, and M. Koch, 2012, Modeling regional sea level rise using local tide gauge data, J. Geod. Sci., Vol. 2, Issue 3, pp. 188-1999.

İz H.B., 2006, How do Unmodeled Systematic MSL Variations Affect Long Term Sea Level Trend Estimates from Tide Gauge Data? Journal of Geodesy, Vol. 80, No .1, pp. 40-46. 
Jevrejeva, S., A. Grinsted, J. C. Moore, and S. Holgate, 2006, Nonlinear trends and multiyear cycles in sea level records, J. Geophys. Res., 111, C09012, doi:10.1029/2005JC003229.

Keeling, C. D., and T. P. Whorf, 1997, Possible forcing of global temperature by oceanic tides. Proc. Natl. Acad. Sci., 94, 83218328.

Van Deemter, K., 2010, Not Exactly: In Praise of Vagueness, Oxford University Press, pp. 341.

Munk, W., M. Dzieciuch, S. Jayne, 2002, Millennial Climate Variability: Is There a Tidal Connection? J. Climate, 15, 370-385.

Permanent Service for Mean Sea Level (PSMSL), 2016, Tide Gauge Data, Retrieved August 2020 from http://www.psmsl.org/data/obtaining/.

Solow, A. R., 1987, Testing for Climate Change: An Application of the Two-Phase Regression Model, J. Clim. Appl. Meteorol. 26, 1401-1405.

Solow, A. R., 1990, Discriminating between Models: An Application to Relative Sea Level at Brest. J. Climate, 3, 792-796.

Woodworth, P.L., Menéndez, M., Gehrels, W.R., 2011, Evidence for Century-Timescale Acceleration in Mean Sea Levels and for Recent Changes in Extreme Sea Levels, Surveys in Geophysics, Vol. 32, No. 4-5, pp. 603-618.

Woodworth, P.L., 1990, A search for accelerations in records of European mean sea-level. Int. J. of Climatology, Vol.,10 (??), pp. 129-143.

Wöppelmann, G., Pouvreau, N., Coulomb, A., Simon, B., Woodworth, P., 2008, Tide gauge datum continuity at Brest since 1711: France's longest sea-level record, Geoph. Res. Lett. Vol. 35.

Wöppelmann, G., Pouvreau, N., Simon, B., 2006, Brest sea level record: A time series construction back to the early eighteenth century, Ocean Dyn., 56, 487 - 497.

Yndestad H., 2006, The influence of the lunar nodal cycle on Arctic climate. ICES. Journal of Marine Science, 63: $401-420$.

Yndestad H., W.R. Turrell, V. Ozhigin, 2008, Lunar nodal tide effects on variability of sea level, temperature, and salinity in the Faroe-Shetland Channel and the Barents Sea, Deep Sea Research Part I: Oceanographic Research Papers, Vol. 55, Issue 10, October 2008, pp 1201-1217. 